\title{
Actitudes ante situaciones de agravio. Un estudio comparativo entre oficiales de fuerzas de seguridad y estudiantes universitarios"
}

\author{
Attitudes towards offenders. Comparative study between \\ security force officials and university students
}

Recibido: febrero 20 de 2009 | Revisado: septiembre 1 de 2009 | Aceptado: octubre 14 de 2009

\author{
SOLANGE RODRÍGUEZ ESPÍNOLA ** \\ José EDUARDO MORENO \\ Consejo Nacional de Investigaciones Científicas \\ y Técnicas (CONICET), Argentina
}

SICI: 1657-9267(201108) 10:2<371:ASAUEC>2.0.TX;2-E

Para citar este artículo. Rodríguez, E. S. \& Moreno, J. E. (2011). Actitudes ante situaciones de agravio. Un estudio comparativo entre oficiales de fuerzas de seguridad y estudiantes universitarios. Universitas Psychologica, 10 (2), 371-380.

Artículo de investigación.

** Centro Interdisciplinario de Investigaciones en Psicología Matemática y Experimental (CIIPME), del Consejo Nacional de Investigaciones Científicas y Técnicas (CONICET). Tte. Gral. Perón 2158, Ciudad Autónoma de Buenos Aires, Código postal 1040, Argentina. E-mail: solange.rodriguezespindola@gmail.com; jemoreno1@yahoo.com Research-ID: Rodríguez, S. E., E-2413-2011.
RES UMEN

Se presenta un estudio comparativo entre personal de fuerzas de seguridad de la Argentina y estudiantes universitarios de Psicología y Abogacía acerca de las actitudes ante situaciones de agravio. La muestra comprendió a 476 sujetos. Se realizaron análisis multivariados de varianza y se observó que los oficiales de Policía obtuvieron medias inferiores en las actitudes agresivas ( $p=0.0001)$ y prosociales $(p=0.0001)$ y un promedio mayor en la dimensión pasividad $(p=0.0001)$. El incremento en cuanto a las actitudes pasivas y la menor puntuación en las actitudes agresivas en los oficiales, se pueden atribuir al fuerte énfasis en la obediencia y el control de la agresividad que la Institución promueve actualmente y que está presente en la formación. Palabras clave autores

Actitudes, agresividad, prosocialidad, Policía.

Palabras clave descriptores

Psicología social, comportamiento, comportamiento social, comportamiento de grupo, análisis de variancia.

\section{A B S T R A C T}

We present a comparative research about attitudes towards offenders between security force officials from Argentina and Psychology and Law university students. The sample comprises 476 participants. We carried out a multivariate analyses of variance and it was observed that policemen got a lower media in aggressive attitudes $(p=0.0001)$ and in prosocial attitudes $(p=0.0001)$, and a higher media in passive attitudes $(p=0.0001)$. The security force officials' increase in passive attitudes and the lower scoring in aggressive attitudes, can be attributed to the strong emphasis in the obedience and aggressiveness control that the institution promotes, and it is present in the police trainning course.

Key words authors

Attitudes, Aggressiveness, Prosocialness, Police.

Key words plus

Social Psychology, Behaviour, Social Behaviour, Group Behaviour, Variance Analysis. 


\section{Introducción}

El desarrollo de actitudes y conductas prosociales es de urgente actualidad en un mundo signado por diversas formas de violencia y por la escasez de conductas solidarias. Tanto el incremento de la criminalidad y del grado de violencia en el actuar delictivo y también en la vida cotidiana de todos los ciudadanos, como la persistencia de excesos y abusos policiales, de no hacer un uso adecuado y proporcional de la fuerza, caracterizan a nuestra América Latina. Cabe señalar que la Policía y las demás fuerzas de seguridad no tienen una política autónoma, sino que cumplen con una tarea estatal de control social, mediante el ejercicio legal de la violencia, del poder de represión.

Debemos tener en cuenta que el actuar policial es un componente fundamental para garantizar el Estado de derecho, que la institución policial es una de las encargadas de hacer cumplir la ley y que la democracia es un sistema político complejo que se sustenta, entre otros pilares, en el respeto pleno de los derechos humanos. Lo primero que debe tener en cuenta un Policía, es que el presunto delincuente es una persona y un ciudadano y que como tal posee derechos humanos que les son inherentes y que merecen respeto. Asimismo, si bien es cierto que deben ser cuidadosos al limitar el derecho a la libertad de las personas, no podemos olvidar que también su función es preservar el derecho de la colectividad, es decir defender los derechos de todos los ciudadanos.

La idea de los mandos policiales, en la mayoría de los países latinoamericanos, es recuperar la imagen en general deteriorada de la Policía y actuar en el marco del pleno respeto a los derechos humanos, para de este modo merecer la aceptación de la ciudadanía. Así, en una muestra de estudiantes de Abogacía en México, la mayoría tenía una evaluación negativa de las fuerzas policiales, siendo la Policía municipal la que tenía peor imagen, luego la estatal y finalmente con mejor imagen aunque negativa, la federal (Brown, Benedict \& Wilkinson, 2006). Debido a esto, la capacitación en derechos humanos ha pasado a ser una parte central de la formación policial. Así señala Aroudo Luis
Estebao, capitán de la Policía militar del estado de Matto Grosso do Sul, Brasil, en el reportaje de T. Barreda: "Nuestro objetivo no es la enseñanza de nuevas técnicas policiales. Nosotros trabajamos para lograr un cambio de actitud, en la mente de los policías, para que los principios de derechos humanos estén presentes en el comportamiento de cada efectivo" (Barreda, 2002, p. 4).

El respeto a la dignidad de las personas no sólo supone el uso proporcional y racional de la fuerza, sino también que el accionar policial debe buscar el trato cortés, el desarrollo de actitudes prosociales, sin que esto sea interpretado como debilidad o falta de autoridad. Se busca un acercamiento con la comunidad, llevando el accionar de la Policía de manera respetuosa, como también eficaz y eficiente, para resolver los múltiples problemas que, sobre seguridad ciudadana, padece la población.

Por este motivo, es importante evaluar en los miembros de esta fuerza de seguridad las actitudes que adoptan cuando reciben un agravio, un daño, por parte de diversas personas y en diversas situaciones. Es decir que la evaluación de estas actitudes permite observar el manejo de la agresividad, su grado de control, así como también la actitud de servicio y de tolerar cierta agresión o daño en función de preservar la armonía social.

\section{Actitudes ante situaciones de agravio}

En la vida estamos expuestos constantemente a situaciones difíciles en las cuales somos agraviados. Existen personas que nos proporcionan sufrimiento, incluso sin desearlo, esto se debe a la condición humana caracterizada por ser frágil, limitada y débil. A veces hacemos el mal incluso pensando hacer el bien.

Las relaciones entre las personas con frecuencia se ven alteradas, lesionadas hasta el punto de su ruptura, debido a transgresiones al código moral que las regulan. Uno de los miembros de una relación es dañado o siente que lo ha sido, emergiendo en él emociones negativas de variada intensidad, que a veces culminan en actos de venganza o en el establecimiento de un sentimiento permanente de rencor y resentimiento. 
A la experiencia de haber sido dañado íntimamente y de sentir herida su dignidad, de ser víctima de una injusticia, sin llegar a las respuestas agresivas antes mencionadas, generalmente la acompañan reclamos de reparación y de justicia. Otros optan por negar la ofensa o someterse al ofensor.

El acto de agravio altera a sus dos protagonistas, víctima y victimario. A veces los encierra en sí mismos y los ata al resentimiento o deseo de venganza (ofendido) y al sentimiento de culpa (ofensor). Otras veces la víctima se convierte en victimario e inicia una secuencia incontenible de agresiones mutuas.

El acto de perdonar permite restaurar la armonía social y las relaciones interpersonales, disminuye el grado de fricción y conflicto social, y es un medio de control social, en el sentido del mantenimiento de relaciones pacíficas. El perdón suscita y promueve sentimientos de amor verdadero. Los sujetos asumen la responsabilidad de cuidar realmente a cada persona. Por eso, un acto dañino por parte de alguien no altera su sentimiento de amor.

En esta investigación se evalúan tres respuestas posibles que pueden dar los sujetos ante las situaciones de agravio y que configuran siete modalidades específicas o actitudes: a) respuestas pasivas: sometimiento y negación; b) respuestas agresivas: reacción hostil, resentimiento y venganza, y c) conductas prosociales: reclamo de explicación y perdón o búsqueda de reconciliación.

En este trabajo, presentamos una investigación comparativa entre personal de fuerzas de seguridad de la Argentina (cadetes de Policía que finalizaban los cursos terciarios de capacitación) y estudiantes universitarios de las carreras de Abogacía y Psicología, para evaluar las diferencias de actitud hacia un ofensor, teniendo en cuenta que son tres profesiones con roles muy diferentes. El rol policial supone una actitud de servicio activa que implica imponer o mantener un orden, el rol del abogado supone un distanciamiento de la situación para poder juzgar de manera imparcial y el rol del psicólogo supone una actitud de servicio, de ayuda mediante la posibilidad de ponerse en el lugar del otro tanto sea víctima o victimario. Además, en el personal policial se espera asertividad y firmeza en su actuar, pero sin caer en excesos en el uso de la fuerza, es decir con un adecuado control de la agresividad.

\section{Estudios previos respecto a esta problemática}

La temática abordada en el presente trabajo no registra exactamente antecedentes en cuanto al estudio de las actitudes ante situaciones de agravio en el ámbito policial, aunque existen numerosos estudios en temas próximos referentes a la agresividad, las conductas prosociales, el estrés, la percepción de situaciones de amenaza y los modos de afrontamiento en policías.

El trabajo policial, y de las fuerzas de seguridad y defensa en general, es considerado como estresante; es descrito como una de las ocupaciones más estresantes en el mundo (Anshel, 2000). Así, Berg, Hem, Lau y Ekeberg (2006) estudiaron en la Policía noruega el estrés laboral y su repercusión en la salud física y mental; encontraron puntajes inferiores de síntomas depresivos y ansiedad en comparación con la población general, pero reportan la existencia de mayores dolores osteomusculares en los policías en cuanto a lo corporal, y de síntomas de despersonalización en lo psicológico.

Herrera y Guarino (2007) han encontrado, en cadetes navales venezolanos, que el uso de modos de afrontamiento poco adaptativos se asocian con el reporte de estrés y con la salud percibida. Además, observaron que los puntajes obtenidos en las dimensiones de frecuencia e intensidad del estrés sugieren que perciben muchas situaciones estresantes, pero que las mismas les generan poco malestar.

Respecto a las conductas prosociales, Adebayo (2005) encontró en oficiales de la Policía de Nigeria que éstas y las actitudes éticas se correlacionan positivamente cuanto mayor es la percepción de tener apoyo organizacional de la Fuerza, como también reconocimiento público.

\section{Método}

La presente investigación es de tipo descriptivocomparativo, con un diseño de corte transversal, 
es decir que las variables fueron evaluadas en su totalidad simultáneamente.

\section{Participantes}

El total de la muestra estuvo conformado por 476 participantes de ambos sexos: 197 varones (41,4 \%) y 279 mujeres (58,6 \%). La composición por carrera fue de 147 alumnos de Psicología, 22 varones $(15 \%)$ y 125 mujeres $(85 \%) ; 118$ estudiantes de Abogacía, 53 varones (44,9\%) y 65 mujeres (55,1 $\%)$; 211 cadetes de las fuerzas de seguridad, 122 varones $(57,8 \%)$ y 89 mujeres (42,2\%). Las edades de los participantes estuvieron comprendidas entre los 18 y 31 años $(M=21,6$ años; $D T=3,81)$. La submuestra de cadetes policiales comprendió a la totalidad de los que estaban concluyendo su período de formación anual en la Escuela de Formación Policial de la localidad de Benavides, perteneciente a la Provincia de Buenos Aires (Argentina). La submuestra de estudiantes universitarios de Psicología y Abogacía comprendió a la totalidad de alumnos de tercer año de una universidad privada confesional de la Provincia de Entre Ríos (Argentina).

\section{Instrumentos}

El Cuestionario de Actitudes ante Situaciones de Agravio (CASA) es una prueba de evaluación de actitudes ante las ofensas (Moreno \& Pereyra, 1999, 2000, 2004; Pereyra \& Moreno, 2001). La prueba consiste en diez relatos de situaciones ofensivas pertenecientes a cinco ámbitos diferentes: trabajo, pareja, padres, amistad y Dios. Cada relato contiene siete ítems referidos a cada una de las escalas previstas: Negación, Sometimiento, Venganza, Hostilidad, Rencor, Reconciliación y Explicación; a su vez estas escalas se agrupan en tres factores: Agresivo, Pasivo y Prosocial. El sujeto debe identificarse con la víctima del relato y responder a cada una de las cuatro alternativas, a saber: siempre, frecuentemente, casi nunca o nunca.

Las siete escalas del CASA se definen del siguiente modo:
1) Respuestas pasivas

I) Sometimiento (S): actitud de subordinación del juicio, decisión o afectos propios a los del ofensor, mediante justificaciones humillantes.

II) Negación del agravio (N): predisposición a modificar en su fantasía situaciones reales de agravio, transformándolas en otras que le resulten tolerables o placenteras.

2) Respuestas agresivas

III) Venganza o Retaliación (V): predisposición a actuar cavilosa y planificadamente para encontrar una satisfacción o desquite ante un agravio u ofensa (venganza) o castigar en un grado similar a lo padecido o sufrido a causa de dicha ofensa. Retaliación: ley del "ojo por ojo, diente por diente".

IV) Rencor y Resentimiento (R): sentimientos de enojo y odio que se guardan interiormente y predisponen a la enemistad o el ensañamiento con el ofensor.

V) Hostilidad (H): predisposición a responder inmediatamente acometiendo o dañando al ofensor.

3) Respuestas prosociales

VI) Demanda de Explicación o Reivindicación (E): actitud de reclamar al ofensor justificaciones y motivos que expliquen su proceder, o exigir recuperar o reparar, total o parcialmente, lo que le dañó o quitó.

VII) Perdón y Búsqueda de Reconciliación (P): actitud de cuidar realmente el vínculo de afecto o amor con los demás, por lo cual un agravio no altera su sentimiento de amor hacia el otro. Permite mantener abierta la posibilidad de la reconciliación. Exige el arrepentimiento y cambio de proceder del otro y cierra las puertas a las acciones de venganza.

En cuanto a las propiedades psicométricas del CASA (Moreno \& Pereyra, 2004), los resultados obtenidos respecto de la confiabilidad como consistencia interna son satisfactorios, con alfas de Cronbach de 0.56 para la escala de Sometimiento, 0.70 para la escala de Negación, 0.77 para Ven- 
ganza, 0.78 para la escala de Rencor, 0.79 para la escala de Hostilidad, 0.64 para Explicación y 0.65 para la escala de Perdón.

Se realizaron estudios factoriales para la validación del constructo mediante el método de ejes principales y de rotación Varimax y Promax, a una muestra de 801 sujetos. Se encontraron siete factores de primer orden correspondientes a las siete escalas con cargas factoriales satisfactorias en prácticamente todos los ítems. Los estudios factoriales de las escalas pusieron en evidencia la existencia de tres factores de segundo orden. Además, se han realizados varios estudios de validez convergente con otros instrumentos, como por ejemplo, con el Cuestionario de Valores Interpersonales de L. Gordon (1977). Se hallaron, por ejemplo, correlaciones negativas significativas entre la escala de Benevolencia de Gordon y las escalas del factor agresión del CASA.

Los estudios de validación (confiabilidad y validez) arrojaron resultados satisfactorios.

\section{Resultados}

Se realizaron análisis multivariados de varianza (MANOVA) para evaluar las diferencias en las dimensiones y escalas del CASA entre las muestras del personal de seguridad (policías) y los estudiantes de las carreras de Abogacía y Psicología.

Respecto de las dimensiones del CASA (véase, Tabla 1) se obtuvo un F de Hotelling ${ }_{(6,970)}=37,45$ con un $p=0.0001$, es decir que globalmente en estas tres dimensiones difieren significativamente los sujetos de estas tres carreras. No fue significativo el efecto de la variable sexo $(p=0.10)$ como tampoco la interacción entre sexo y carrera $(p=0.16)$.

Analizando los $F$ univariados, también se puede observar (véase Tabla 1) que, tomadas por separado, existen diferencias significativas en cada una de las dimensiones, a saber: en las actitudes agresivas hacia un ofensor $(p=0.0001)$, prosociales $(p=$ $0.0001)$ y en la dimensión pasividad $(p=0.0001)$. Se realizaron finalmente contrastes multivariados de los que resultó que:

a) En la dimensión Pasiva la media de los policías es significativamente mayor tanto respecto a los alumnos de Abogacía ( $p=0.02$ ) como a los de Psicología ( $p=0.0001)$, y también es significativamente mayor el promedio de los alumnos de Abogacía respecto de los de Psicología ( $p=$

TABLA 1

Medias y desvíos típicos de las dimensiones del CASA según carrera

\begin{tabular}{llccccc}
\hline $\begin{array}{c}\text { Dimensiones del } \\
\text { CASA }\end{array}$ & \multicolumn{1}{c}{ Carrera } & Media & $\begin{array}{c}\text { Desvío } \\
\text { Típico }\end{array}$ & $n$ & $F$ & $p=$ \\
\hline \multirow{3}{*}{ Pasiva } & Psicología & 2.22 & 0.31 & 147 & & \\
& Abogacía & 2.37 & 0.34 & 118 & 24.41 & 0.0001 \\
& Fuerzas de Seguridad & 2.48 & 0.39 & 211 & & \\
\multirow{2}{*}{ Agresiva } & Psicología & 1.93 & 0.38 & 147 & & \\
& Abogacía & 2.11 & 0.45 & 118 & 75.48 & 0.0001 \\
& Fuerzas de Seguridad & 1.58 & 0.37 & 211 & & \\
\multirow{3}{*}{ Prosocial } & Psicología & 3.18 & 0.32 & 147 & & \\
& Abogacía & 3.04 & 0.40 & 118 & 11.83 & 0.0001 \\
& Fuerzas de Seguridad & 2.99 & 0.35 & 211 & & \\
\hline
\end{tabular}

Fuente: elaboración propia. 
0.002). Es decir que los cuatro contrastes fueron significativos respecto a la dimensión Pasiva.

b) En la dimensión Agresiva la media de los policías es significativamente menor tanto respecto a los alumnos de Abogacía ( $p=0.0001)$ como a los de Psicología $(p=0.0001)$, y es significativamente mayor el promedio de los alumnos de Abogacía respecto de los de Psicología ( $p=$ 0.001). Es decir que los cuatro contrastes fueron significativos respecto a la dimensión Agresiva.

c) En la dimensión Prosocial, las medias de los policías es significativamente menor respecto a los alumnos de Psicología $(p=0.03)$ pero no respecto de los de Abogacía $(p=0.86)$, y también es significativamente menor el promedio de los alumnos de Abogacía respecto de los de Psicología $(p=0.005)$. Es decir que tres contrastes fueron significativos respecto a la dimensión Prosocial.

En el Gráfico 1, se pueden observar las diferencias de las dimensiones del CASA entre las tres carreras.

\section{GRÁfICO 1}

Dimensiones del CASA según carrera.
Respecto de las escalas del CASA se obtuvo un $F$ de Hotelling $_{(14,932)}=18,65$ con un $p=$ 0.0001 , es decir que globalmente en estas siete escalas difieren significativamente los sujetos de estas tres carreras. Analizando los F univariados se puede observar (Tabla 2) también que, tomadas por separado, existen diferencias significativas en cada una de las escalas, a saber: en las actitudes de Sometimiento hacia un ofensor $(p=0.002)$, Negación de la ofensa $(\mathrm{p}=0.0001)$, Venganza $(p=0.0001)$, Rencor $(p=0.0001)$, Hostilidad $(p=0.0001)$, Explicación $(p=0.0001)$ y Perdón $(p=0.009)$.

Se realizaron finalmente contrastes multivariados de los que resultaron significativos:

a) En cuanto a la escala de Sometimiento, los policías tienen una actitud mayor de sometimiento que los alumnos de Psicología ( $p=0.001$ ).

b) Los policías tienden a negar la ofensa en mayor medida que los alumnos de Psicología $(p=$ 0.0001) y de Abogacía ( $p=0.01)$. Asimismo,

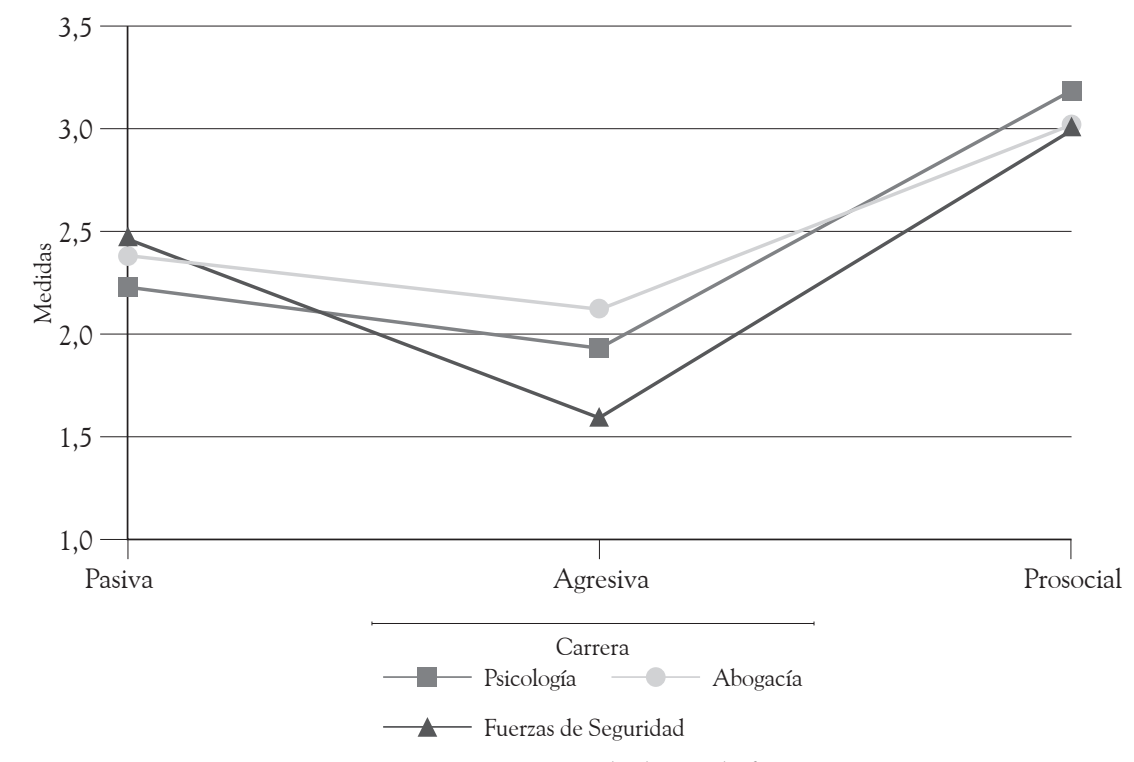

Dimenciones. Actitudes hacia el ofensor

Fuente: elaboración propia. 
TABLA 2

Medias y desvíos típicos de las escalas del CASA según carrera

\begin{tabular}{|c|c|c|c|c|c|c|}
\hline Escalas del CASA & Carrera & Media & $\begin{array}{l}\text { Desvío } \\
\text { Típico }\end{array}$ & $n$ & $F$ & $p=$ \\
\hline \multirow{3}{*}{ Sometimiento } & Psicología & 2.35 & 0.37 & 147 & \multirow{3}{*}{6.43} & \multirow{3}{*}{0.002} \\
\hline & Abogacía & 2.42 & 0.34 & 118 & & \\
\hline & Fuerzas de Seguridad & 2.51 & 0.44 & 211 & & \\
\hline \multirow{3}{*}{ Negación } & Psicología & 2.08 & 0.35 & 147 & \multirow{3}{*}{32.72} & \multirow{3}{*}{0.0001} \\
\hline & Abogacía & 2.31 & 0.45 & 118 & & \\
\hline & Fuerzas de Seguridad & 2.45 & 0.43 & 211 & & \\
\hline \multirow{3}{*}{ Venganza } & Psicología & 1.47 & 0.38 & 147 & \multirow{3}{*}{56.95} & \multirow{3}{*}{0.0001} \\
\hline & Abogacía & 1.78 & 0.53 & 118 & & \\
\hline & Fuerzas de Seguridad & 1.29 & 0.32 & 211 & & \\
\hline \multirow{3}{*}{ Rencor } & Psicología & 2.08 & 0.45 & 147 & \multirow{3}{*}{48.11} & \multirow{3}{*}{0.0001} \\
\hline & Abogacía & 2.21 & 0.53 & 118 & & \\
\hline & Fuerzas de Seguridad & 1.72 & 0.46 & 211 & & \\
\hline \multirow{3}{*}{ Hostilidad } & Psicología & 2.24 & 0.46 & 147 & \multirow{3}{*}{73.37} & \multirow{3}{*}{0.0001} \\
\hline & Abogacía & 2.33 & 0.52 & 118 & & \\
\hline & Fuerzas de Seguridad & 1.74 & 0.48 & 211 & & \\
\hline \multirow{3}{*}{ Explicación } & Psicología & 3.12 & 0.38 & 147 & \multirow{3}{*}{17.38} & \multirow{3}{*}{0.0001} \\
\hline & Abogacía & 3.00 & 0.48 & 118 & & \\
\hline & Fuerzas de Seguridad & 2.85 & 0.44 & 211 & & \\
\hline \multirow{3}{*}{ Perdón } & Psicología & 3.23 & 0.36 & 147 & \multirow{3}{*}{4.72} & \multirow{3}{*}{0.009} \\
\hline & Abogacía & 3.08 & 0.46 & 118 & & \\
\hline & Fuerzas de Seguridad & 3.14 & 0.40 & 211 & & \\
\hline
\end{tabular}

Fuente: elaboración propia.

los alumnos de Psicología niegan menos que los de Abogacía $(p=0.0001)$.

c) Los policías tienden a adoptar una actitud menos vengativa ante una ofensa que los alumnos de Psicología $(p=0.0001)$ y de Abogacía $(p=$ 0.0001). Asimismo, los alumnos de Psicología son menos vengativos que los de Abogacía ( $p$ $=0.0001$ ).

d) Los policías tienden a adoptar una actitud menos rencorosa ante una ofensa que los alumnos de Psicología $(p=0.0001)$ y de Abogacía $(p=$ 0.0001).

e) Los policías tienden a adoptar una actitud de menor hostilidad ante una ofensa que los alumnos de Psicología $(p=0.0001)$ y de Abogacía $(p=0.0001)$.

f) Los policías tienden a adoptar una actitud de pedir menos explicaciones ante una ofensa que los alumnos de Psicología $(p=0.0001)$ y de Abogacía $(p=0.01)$. Asimismo, los alumnos 
de Abogacía tienden a adoptar una actitud de pedir menos explicaciones ante una ofensa que los alumnos de Psicología $(p=0.05)$.

g) Por último, los alumnos de Abogacía tienden a adoptar una actitud de perdonar en menor medida que los alumnos de Psicología $(p=0.008)$.

En el Gráfico 2 se pueden observar y comparar los perfiles de las escalas del CASA de las tres carreras.

Dado los resultados obtenidos, se hicieron algunos análisis complementarios para analizar por qué los policías habían obtenido un mayor puntaje en las escalas de Sometimiento y de Negación. Analizando mediante el análisis multivariado de varianza (MANOVA) los puntajes obtenidos en las escalas en cada ámbito, se obtuvo respecto de la escala de Sometimiento que solo en el ámbito del trabajo, los policías obtuvieron diferencias significativas con las otras dos carreras $(p=0.001$ con Psicología y $p=0.04$ con Abogacía). En los demás ámbitos, el nivel de sometimiento al ofensor fue similar a los demás, levemente mayor. Respecto a la escala de Negación no se observan diferencias por ámbito en los policías, y la tendencia a negar la ofensa es mayor para los policías cualquiera sea la situación. Es decir que en la dimensión Pasiva existen diferencias en los policías respecto al Sometimiento y la Negación, según los ámbitos en que se dé la ofensa.

\section{Discusión y conclusiones}

Los resultados de esta investigación brindan información de diferencias en actitudes frente al agravio de estudiantes universitarios de Psicología, Abogacía y cadetes de Policía que están concluyendo la Tecnicatura en Seguridad Pública. Estudios similares no han sido investigados en muestras de fuerzas de seguridad en América Latina.

GRÁFICO 2

Medias de las escalas del CASA según carrera

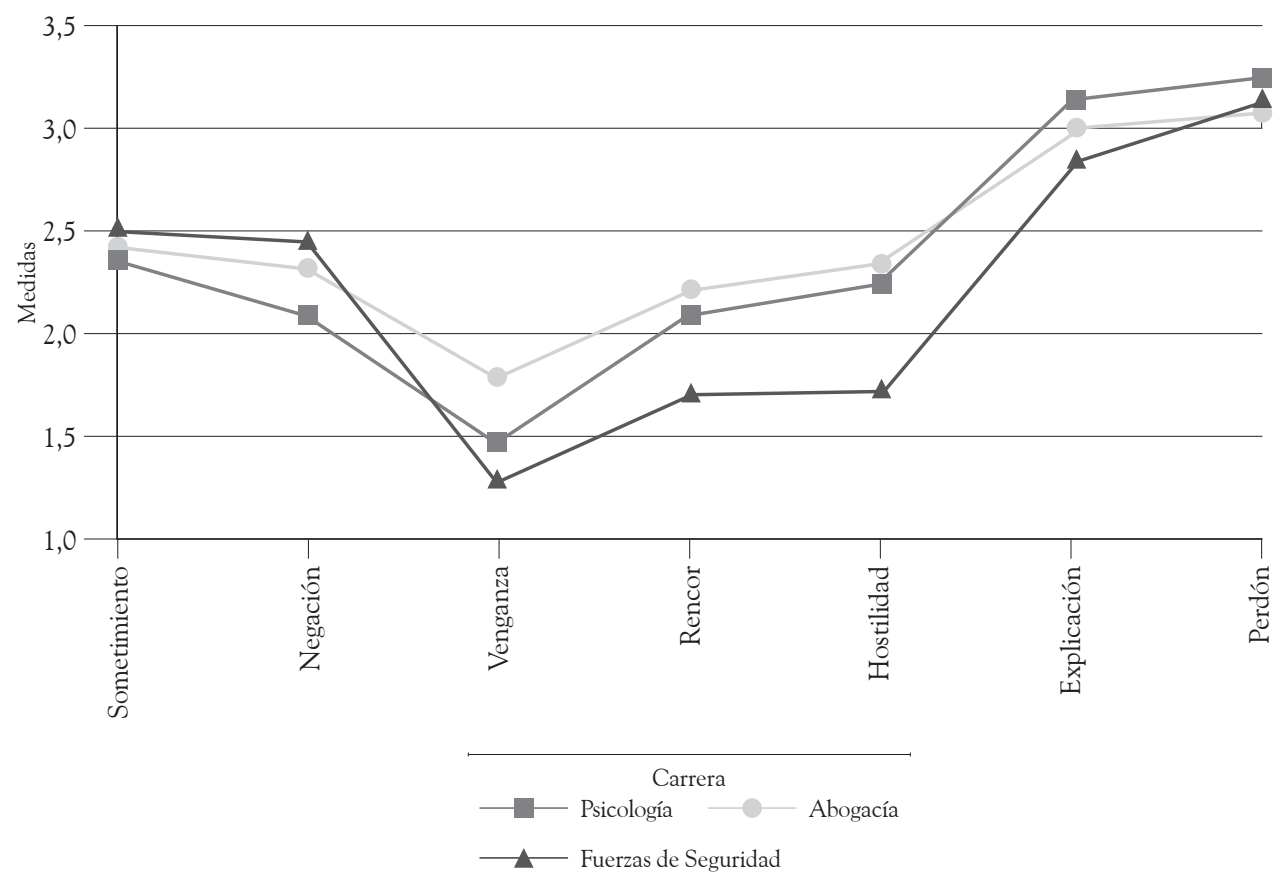

Escalas de Actitudes ante Situaciones de Agravio

Fuente: elaboración propia. 
Como se afirmó en la introducción, el rol policial supone una actitud de servicio activa que implica imponer o mantener un orden, mientras que el rol del abogado supone un distanciamiento de la situación, o una acción más racional que afectiva, para poder juzgar de manera imparcial, y el rol del psicólogo supone una actitud de servicio, de ayuda mediante la posibilidad de ponerse en el lugar del otro, sea víctima o victimario. El incremento en cuanto a las actitudes pasivas y la menor puntuación en las actitudes agresivas en los oficiales de Policía, se puede atribuir al fuerte énfasis en la obediencia y el control de la agresividad que la Institución promueve y que está presente en los cursos de formación que estaban realizando.

Los policías se percibieron con mayor sometimiento, el cual supone, en alguna medida, una actitud de subordinación del juicio, decisión o afectos propios a los del ofensor. Sin embargo, al observarse las diferencias de sometimiento en los distintos ámbitos que evalúa el instrumento, los policías demostraban diferencias con los estudiantes de Psicología y Abogacía en el ámbito laboral. De esta manera, podemos decir que el perfil que quisieron mostrar de sometimiento en el ámbito de trabajo, pudo ser influido por el marco institucional en el que están incluidos, tratando de dar una imagen positiva o, por lo menos, de acuerdo a lo que socialmente se espera o debería ser.

También se observa una mayor predisposición a modificar en su fantasía situaciones reales de agravio, transformándolas en otras que les resultan tolerables o placenteras (negación). El proceso de admisión y permanencia en la formación policial implica el buen manejo de las emociones y un autocontrol frente a la agresión.

Por otra parte, llama la atención que los cadetes de Policía en las conductas prosociales tengan puntuaciones menores que los estudiantes universitarios, dado que el rol policial supone una actitud de servicio a la sociedad. Quizás esto se explique porque el énfasis de la instrucción está puesto principalmente en el autocontrol, la subordinación y el manejo de la agresividad por sobre las conductas de prosocialidad. Además, debido a la necesidad de seguridad frente al delito, las po- líticas de formación policial y la sociedad misma no promueven que los policías posean la cualidad de tener actitudes tales como la de reclamar al ofensor justificaciones y motivos que expliquen su proceder, ni de permitir la posibilidad de la reconciliación con el agresor, que evalúan las escalas prosociales del CASA. Probablemente, en la formación se enfatice el suprimir o reprimir lo que no es considerado correcto, olvidando el promover los valores y actitudes prosociales que naturalmente encauzarían la agresividad sin caer en la pasividad o el sometimiento.

Los alumnos de Psicología se percibieron con actitudes menos pasivas hacia la ofensa, demostrando menor sometimiento y negación. Estas características también podrían estar relacionadas con la formación profesional, debido a que en muchos casos una negación de la situación no sería la respuesta salutogénica esperable desde la salud mental. Respecto a que las actitudes prosociales fueron mayores en la submuestra de alumnos de Psicología, esto podría relacionarse con lo que es esperable socialmente en el desempeño de este rol, ya que serán profesionales que tendrán que ponerse en la situación de entender al otro. La actitud de búsqueda o solicitud de explicación y de perdonar es en esta profesión importante, para lograr un buen vínculo terapéutico y lograr la cura.

Los estudiantes universitarios de Abogacía demostraron tener actitudes más agresivas frente al agravio, percibiéndose con más actitud de venganza, guardando interiormente sentimientos de enojo y odio que predisponen a la enemistad o el ensañamiento con el ofensor, y con más hostilidad. El ejercicio profesional de la Abogacía requiere una cierta expresión de la agresividad, en particular la verbal no la física, mayor que en otras profesiones. Así, el rol de fiscal como el de abogado defensor requieren firmeza y asertividad, no dejarse someter a las opiniones de otros; la pasividad es vista como muy negativa en el desempeño profesional.

Por último, consideramos que la investigación de las actitudes pasivas, agresivas y prosociales de los futuros oficiales, parece ser importante y necesaria para la mejor formación y el desempeño posterior. Además, estas variables deben ser estudiadas 
conjuntamente con los rasgos de personalidad, el juicio moral, el sistema de valores y creencias, entre otras.

\section{Referencias}

Adebayo, D. O. (2005). Ethical attitudes and prosocial behaviour in the Nigeria police: Moderator effects of perceived organizational support and public recognition. Policing: An International Journal of Police Strategies and Management, 28 (4), 684-705.

Anshel, M. H. (2000). A conceptual model and implications for coping with stressful events in police work. Criminal Justice and Behavior, 27, 375-400.

Barreda, T. (2002, junio 17). Perú: Más fuerza, menos violencia: Policía nacional respeta los derechos humanos. El Peruano, Diario Oficial, p. 4.

Berg, A. M., Hem, E., Lau, B. \& Ekeberg, O. (2006, December). An exploration of job stress and health in the Norwegian police service: A cross sectional study. Journal of Occupational Medicine and Toxicology, 1, 26. Recuperado el 20 de febrero de 2009, de http://www.occup-med.com/currentissue/browse. asp date $=$ \&volume $=1 \&$ sort $=$ \&page $=2$

Brown, B., Benedict, W. R. \& Wilkinson, W. V. (2006). Public perceptions of the police in Mexico: A ca- se study. Policing: An International Journal of Police Strategies and Management, 29 (1), 158-175.

Gordon, L. V. (1977) Cuestionario de Valores Interpersonales. Madrid: TEA (J. Forteza, trad.).

Herrera, V. \& Guarino, L. (2007). Sensibilidad emocional, estrés y salud percibida en cadetes navales venezolanos. Universitas Psychologica, 7 (1), 185-198.

Moreno, J. E. \& Pereyra, M. (1999). Manual del Cuestionario de Actitudes ante Situaciones de Agravio. Entre Ríos: Universidad Adventista del Plata.

Moreno, J. E. \& Pereyra, M. (2000). Cuestionario de Actitudes ante Situaciones de Agravio (CASA). Manual. Fundamentación teórica, validación y administración. Libertador San Martín, Entre Ríos: Universidad Adventista del Plata.

Moreno, J. E. \& Pereyra, M. (2004). Cuestionario de Actitudes ante Situaciones de Agravio (CASA). Manual (versión actualizada). Lima: Universidad Peruana Unión.

Pereyra, M. \& Moreno, J. E. (2001). Attitude toward offenders scale. Assessment, validation and research. In M. Martínez (Ed.), Prevention and Control of Aggression and the Impact on its Victims (pp. 377-384). New York: Kluwer Academic/Plenum Publishers. 